PAPER

\title{
Right prosubiculum amyloid plaque density correlates with anosognosia in Alzheimer's disease
}

\author{
G A Marshall, D I Kaufer, O L Lopez, G R Rao, R L Hamilton, S T DeKosky
}

J Neurol Neurosurg Psychiatry 2004;75:1396-1400. doi: 10.1136/jnnp.2003.030007

See end of article for authors' affiliations

\section{Correspondence to:}

Dr D Kaufer, UNC Schoo

of Medicine, CB\# 7025,

3114 Bioinformatics Bldg,

Chapel Hill, NC 27599,

USA; kauferd@glial.med. unc.edu

Received 8 October 2003 Revised 19 December 2003 Accepted

20 December 2003

\begin{abstract}
Background: Anosognosia is a common manifestation of Alzheimer's disease. There is an association between impaired awareness and frontal-executive cognitive deficits. Anosognosia is also correlated with decreased metabolism in the right hemisphere, particularly in frontal lobe regions.

Objective: To investigate pathological correlates of anosognosia in Alzheimer's disease.

Design: 41 subjects followed longitudinally in the University of Pittsburgh memory disorders clinic and with necropsy verified Alzheimer's disease were divided into two groups, based on previous clinical assessment: +Aware $(n=23)$ and - Aware $(n=18)$. A subset analysis matching subjects for dementia severity using mini-mental state examination scores was also carried out (13 +Aware; 13 -Aware). Histopathological data from necropsy brain tissue consisted of senile plaque (SP) and neurofibrillary tangle (NFT) counts (regional density) from four different brain regions in the right and left hemispheres: superior and middle frontal gyri (SMF), superior temporal isocortex (ST), the prosubiculum of the hippocampus (PRO), and the entorhinal cortex (EC).

Results: SP density was greater in the right PRO region of -Aware subjects $(F=6.54, p=0.015)$ than +Aware subjects. Significant differences between SP or NFT density were not observed in any other regions. In the subset analysis matching for dementia severity, SP density was again greater in the right PRO region of - Aware subjects than in the other regions $(F=12.72, p=0.002)$.

Conclusions: Increased SP density in the right PRO region suggests that selective pathological involvement of this area contributes to awareness deficits in Alzheimer's disease. The putative role of the PRO in self appraisal may reflect its interconnections with other medial temporal and prefrontal regions.
\end{abstract}

A lzheimer's disease has many neuropsychiatric manifestations and one of the more clinically significant and common of these is lack of awareness of neurological deficits, or anosognosia. Several studies have investigated clinical, neuropsychological, and functional imaging data in an attempt to localise anosognosia to specific regions or circuits in the brain. In one such study, by Lopez et al, anosognosia was determined by questioning the subject and an informant about awareness of deficits, as well as by observing the subject's state after completing a mental status examination. The results indicated greater deficits in frontal functions or "central executive system" functioning in Alzheimer's disease subjects with anosognosia. Michon et al used neuropsychological data, most notably the Wisconsin card sorting test, and found that the degree of anosognosia was correlated with executive or frontal lobe dysfunction in Alzheimer's disease. ${ }^{2}$ Ott et al noted a typical failure of self monitoring in early Alzheimer's disease, as well as a correlation of awareness of deficits with performance on executive and visuospatial function tests. ${ }^{3}$

Among several studies of anosognosia in Alzheimer's disease reported by Starkstein and colleagues, ${ }^{4-7}$ one involving single photon emission computed tomography (SPECT) imaging showed that anosognosia was associated with decreased regional cerebral blood flow in the right hemisphere, particularly in prefrontal (dorsolateral and orbitofrontal) areas. ${ }^{7}$ In another SPECT study, Reed et al found that anosognosia in Alzheimer's disease was correlated with right dorsolateral frontal lobe (frontal superior area) perfusion deficits. ${ }^{8}$ Ott and colleagues ${ }^{9}$ showed that lack of insight in Alzheimer's disease was associated with right temporooccipital region hypoperfusion. Together, these studies impute a relation between anosognosia in Alzheimer's disease and right hemisphere dysfunction, principally involving frontal and temporal regions.

To our knowledge, there have not been any clinicopathological studies of anosognosia in Alzheimer's disease. In the current study we examined senile plaque (SP) and neurofibrillary tangle (NFT) counts, used as correlates of disease burden, from frontal and temporal lobe regions in both hemispheres to investigate possible neuropathological substrates of anosognosia in Alzheimer's disease. Based on previous clinical and neuroimaging studies, we hypothesised that one or more of the right hemisphere regions would have a significantly greater SP or NFT burden, or both, in Alzheimer's disease subjects with impaired awareness of deficits than in subjects with relatively preserved awareness.

\section{METHODS}

\section{Study population}

Subject selection criteria included necropsy verified Alzheimer's disease and the exclusion of other significant and potentially confounding pathological diagnoses such as other neurodegenerative diseases and dementias (subjects with vascular dementia were specifically excluded). The subjects had been followed longitudinally in the University of Pittsburgh Alzheimer's Disease Research Center (ADRC) memory disorders clinic. All subjects had one or more comprehensive assessments, including an evaluation by a neurologist of the subject's degree of awareness (assessment of performance) after completion of neuropsychological testing. Among 224 subjects with neuropathological data who were initially reviewed, 104 with Alzheimer's disease (excluding other significant pathological concomitants) and complete neuropathological data were identified. A subsequent review of the clinical data yielded 41 subjects with both 
Table 1 Sex, age, duration of symptoms, interval between last examination and death, and mini-mental state examination score in subjects with and without anosognosia

\begin{tabular}{llllll}
\hline & Sex $(M / F)$ & Age (years) & $\begin{array}{l}\text { Duration } \\
\text { (years) }\end{array}$ & $\begin{array}{l}\text { Examination to death } \\
\text { interval (years) }\end{array}$ & MMSE score \\
\hline +Aware $(n=23)$ & $11 / 12$ & $79(8)$ & $8(5)$ & $2(1)$ & $14(7) \dagger$ \\
- Aware $(n=18)$ & $11 / 7$ & $76(9)$ & $7(4)$ & $2(1)$ & $9(7) \dagger$ \\
+Aware $(n=13)^{*}$ & $6 / 7$ & $79(9)$ & $8(3)$ & $2(1)$ & $10(3)$ \\
-Aware $(n=13)^{*}$ & $7 / 6$ & $77(9)$ & $7(3)$ & $2(1)$ & $9(4)$ \\
\hline
\end{tabular}

neuropathological and clinical data who died between 1987 and 1992. Subjects were matched for sex, age, duration of symptoms, and interval between the last examination and death (table 1).

\section{Clinical data}

After the standard ADRC evaluation, which included a comprehensive mental status examination, subjects were classified with respect to demonstrable awareness of their own cognitive deficits by the examining clinician on an informal semiquantitative scale:

1. Spontaneous complaint.

2. Agrees when questioned.

3. Unaware.

4. Denies but appears aware.

This rating was based on information obtained during a clinical interview by the examiner in conjunction with formal neuropsychological testing. For purposes of the current study, subjects were divided into +Aware (without anosognosia, categories 1 and 2), and -Aware (with anosognosia, categories 3 and 4). Ratings used for analyses were those from the last clinical visit before death. Of 41 subjects, 23 were +Aware and 18 were -Aware. As dementia severity, based on mini-mental state examination (MMSE) ${ }^{10}$ scores, was significantly greater in -Aware subjects, a subset of 26 subjects was matched for dementia severity (13+Aware and 13 -Aware, table 1) to eliminate this potential confounder.

\section{Neuropathological data}

Brain removal, dissection, tissue sampling, fixation, processing, staining, and SP and NFT counting using Bielschowsky stain have been described previously. ${ }^{11}{ }^{12}$ SP and NFT counts were done by two neuropathologists separately, and a third neuropathologist verified the Alzheimer's disease diagnosis and resolved differences in the counts. Senile plaque counts included both neuritic and diffuse plaques, and the neuropathological diagnosis of Alzheimer's disease was based on Khachaturian criteria, ${ }^{13}$ which were the prevailing criteria at the time of pathological examination. Although more recent NIA-Reagan criteria ${ }^{14}$ include probabilistic diagnostic criteria based on NFT and SP counts, virtually all subjects included in this study would meet criteria for "intermediate" or "high" probability of Alzheimer's disease.

Five microscopic fields were counted between cortical layers II and IV of the different brain regions at a magnification of $\times 200$, and the mean SP and NFT counts were calculated for each region. Neuropathological raters were blind to awareness group. Pearson correlation coefficients for repeated rater and inter-rater reliability for both counts were high (for SP counts, 0.94 and 0.97, respectively; for NFT counts, 0.87 and 0.91 , respectively). ${ }^{12}$ Counts were obtained from the following right and left brain regions: superior and middle frontal gyri (SMF), superior temporal isocortex (ST), prosubiculum of the hippocampus (PRO), and entorhinal cortex (EC). The regions selected for analysis were based on previous unrelated neuropathological studies ${ }^{11} 12$ and therefore do not necessarily represent a comprehensive assessment for pathological substrates of anosognosia. Analysis of variance (ANOVA) was used to compare SP and NFT counts in left and right homologous regions to test the hypothesis that -Aware subjects would have greater SP and/ or NFT burden in right frontal and temporal regions than +Aware subjects.

\section{RESULTS}

In the initial group of 41 subjects analysed (+Aware, $n=23$; -Aware, $\mathrm{n}=18$ ), SP density was significantly greater only in the right PRO region of -Aware subjects $(F=6.54, p=0.015$; table 2). Comparison of the other regions in each hemisphere showed no differences between +Aware and - Aware subjects on SP, NFT, or combined SP+NFT density. A $\chi^{2}$ analysis for sex by group showed no significant difference.

In the subset analysis of 26 subjects (+Aware, $\mathrm{n}=13$; - Aware, $\mathrm{n}=13$ ) matched for dementia severity using MMSE scores, SP density was again significantly greater in the right PRO region of -Aware subjects than in +Aware subjects $(\mathrm{F}=12.72 ; \mathrm{p}=0.002)$. SP density also showed a borderline significant trend towards being higher in the left PRO region of -Aware subjects compared with + Aware subjects $(\mathrm{F}=4.23, \mathrm{p}=0.051)$. No differences in NFT density or combined SP+NFT density were observed in any other region between +Aware and -Aware subjects in this derivative sample. After Bonferroni correction for multiple comparisons (four regions $\times$ two sides $\times$ two measures), the finding of increased SP density in the right $\mathrm{PRO}$ region remained significant $(\mathrm{p}=0.032)$.

\section{DISCUSSION}

Using necropsy data from a retrospective case series, we examined a hypothesis based on clinical studies that anosognosia in subjects with Alzheimer's disease would be associated with greater pathological involvement of right frontal or temporal regions. The principal finding of greater SP density in the right prosubiculum region, which was statistically robust after controlling for dementia severity and multiple comparisons, is consistent with the main study hypothesis and is to our knowledge the first report of a neuropathological correlate of anosognosia in Alzheimer's disease. Although this retrospective study must be considered preliminary, owing to various methodological limitations, it provides insight into the putative anatomical substrates of impaired awareness in Alzheimer's disease.

Anatomically, the PRO region of the hippocampal formation was originally recognised by Lorente de Nó $^{15}$ as the 
Table 2 Senile plaque and neurofibrillary tangle counts in four brain regions

\begin{tabular}{|c|c|c|c|c|c|}
\hline & & + Aware $(n=23)$ & -Aware $(n=18)$ & + Aware $(n=13)^{*}$ & -Aware $(n=13)^{*}$ \\
\hline SMF & $\begin{array}{l}\text { R SP } \\
\text { R NFT } \\
\text { L SP } \\
\text { L NFT }\end{array}$ & $\begin{array}{l}23(19) \\
6(7) \\
22(17) \\
7(7)\end{array}$ & $\begin{array}{l}21(13) \\
8(7) \\
20(12) \\
9(8)\end{array}$ & $\begin{array}{l}20(11) \\
7(6) \\
19(11) \\
9(7)\end{array}$ & $\begin{array}{l}22(14) \\
7(6) \\
22(13) \\
9(9)\end{array}$ \\
\hline ST & $\begin{array}{l}\text { R SP } \\
\text { R NFT } \\
\text { L SP } \\
\text { L NFT }\end{array}$ & $\begin{array}{l}20(17) \\
9(10) \\
22(21) \\
9(9)\end{array}$ & $\begin{array}{l}17(12) \\
11(10) \\
23(22) \\
12(10)\end{array}$ & $\begin{array}{l}18(8) \\
10(9) \\
20(16) \\
10(9)\end{array}$ & $\begin{array}{l}15(9) \\
10(10) \\
25(25) \\
11(10)\end{array}$ \\
\hline PRO & $\begin{array}{l}\text { R SP } \\
\text { R NFT } \\
\text { L SP } \\
\text { L NFT }\end{array}$ & $\begin{array}{l}7(4) \dagger \\
19(16) \\
9(4) \\
23(17)\end{array}$ & $\begin{array}{l}11(6) \dagger \\
24(15) \\
12(6) \\
28(16)\end{array}$ & $\begin{array}{l}6(4) \ddagger \\
22(19) \\
8(4) \S \\
25(20)\end{array}$ & $\begin{array}{l}12(4) \ddagger \\
28(14) \\
12(6) \S \\
33(15)\end{array}$ \\
\hline EC & $\begin{array}{l}\text { R SP } \\
\text { R NFT } \\
\text { L SP } \\
\text { L NFT }\end{array}$ & $\begin{array}{l}16(9) \\
15(8) \\
15(11) \\
15(8)\end{array}$ & $\begin{array}{l}17(10) \\
16(9) \\
15(10) \\
16(8)\end{array}$ & $\begin{array}{l}16(11) \\
15(8) \\
16(14) \\
15(8)\end{array}$ & $\begin{array}{l}16(11) \\
15(9) \\
12(7) \\
16(7)\end{array}$ \\
\hline \multicolumn{6}{|c|}{$\begin{array}{l}\text { Values are means (SD). } \\
\text { *Subset matched for dementia severity. } \\
t p=0.015 ; \neq p=0.002 ; \xi p=0.051 \text {. } \\
\text { EC, entorhinal cortex; L, left; NFT, neurofibrillary tangle; PRO, prosubiculum of the hippocampus; R, right; SMF, } \\
\text { superior and middlle frontal gyri; ST, superior temporal isocortex; SP, senile plaque; +Aware, without anosognosia; } \\
\text {-Aware, with anosognosia. }\end{array}$} \\
\hline
\end{tabular}

transition zone between the subiculum and the adjacent CAl region of the hippocampus (fig 1 ). This nomenclature has not been universally accepted, in part because the PRO region is not well developed in rodents. The phylogenetic trend towards a more differentiated zone of transitional cortex between the CAl and subicular regions of the hippocampal formation may augur a role for the PRO in integrative cognitive processes such as gnosis.

Hyman and colleagues ${ }^{16}$ drew attention to the prosubiculum as being one of the subregions of the hippocampal formation consistently bearing the greatest burden of plaque and tangle pathology in Alzheimer's disease. Anatomically, the prosubiculum is a primary source of hippocampal formation efferents from the intrinsic perforant pathway originating in layer II of the entorhinal cortex to the amygdala (particularly the accessory basal nucleus) and to layer IV of the entorhinal cortex (fig 2). Both the entorhinal cortex and the prosubiculum receive reciprocal projections from the amygdala, which has been postulated to play a

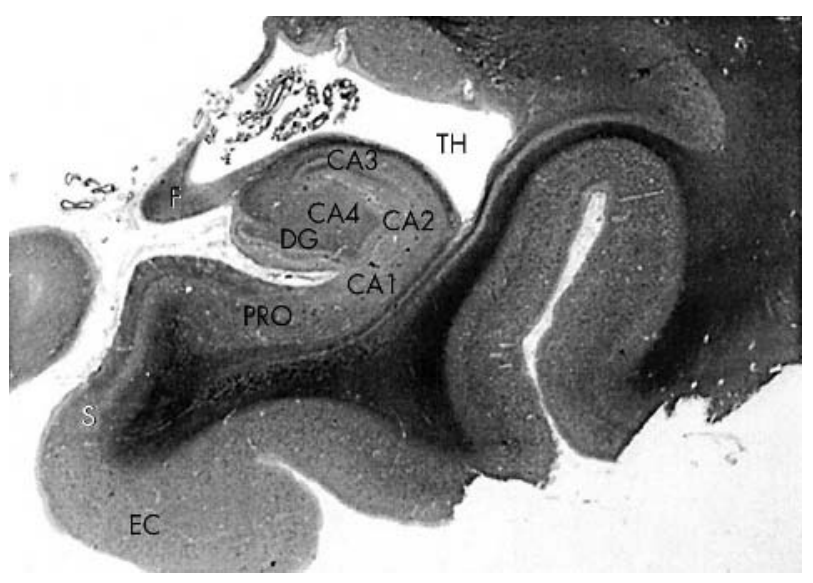

Figure 1 Transverse section through the hippocampus and adjacent regions. CA1, CA2, CA3, CA4, regions of the hippocampus; DG, dentate gyrus; EC, entorhinal cortex; F, fimbria; PRO, prosubiculum; $S$, subiculum; TH, temporal horn of the lateral ventricle. Magnification $\times 8$. functional role in conscious awareness. ${ }^{17}$ The connectivity of the amygdala suggests that information regarding emotional valence supporting awareness may be conveyed to the hippocampal formation primarily at the input and output zones of its intrinsic circuitry. The presence of feedback projections from the prosubiculum to layer IV of the entorhinal cortex, which is typically less severely involved than layer II in Alzheimer's disease, suggest that pathological involvement of the deeper layers of the entorhinal cortex may

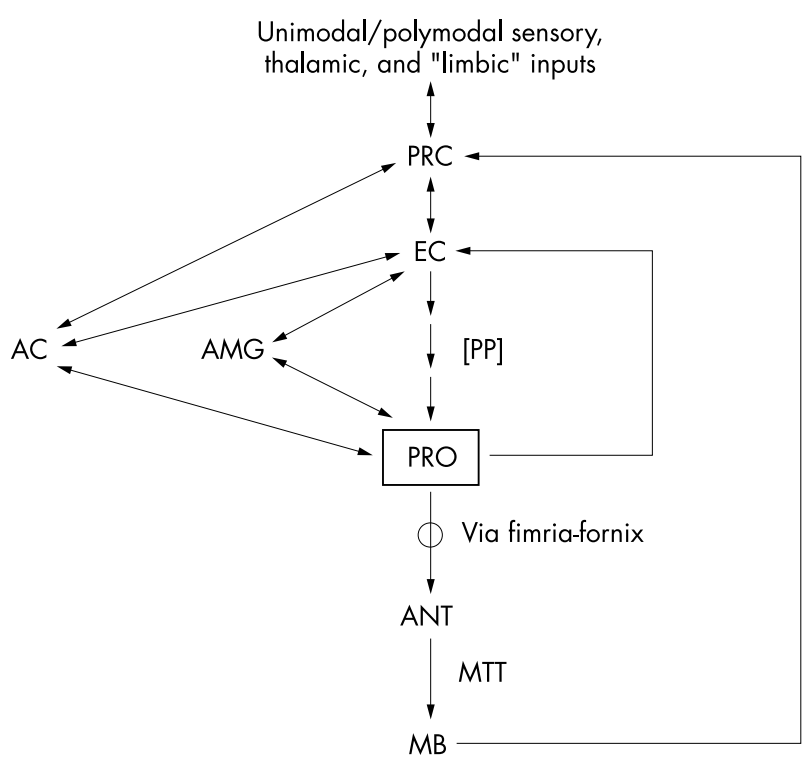

Figure 2 Principal connections of the prosubiculum. Pathways on the right side of the figure include feedback projections from the prosubiculum (PRO) to the entorhinal cortex (EC), and output to diencephalic structures (MB, mammillary body; ANT, anterior nucleus of the thalamus) that are implicated in episodic memory. The left side of the figure depicts connections from the PRO to the amygdala (AMG) and anterior cingulate $(\mathrm{AC})$ that are putatively implicated in self awareness. $M T$, mammilothalamic tract; PP, perforant pathway, the unidirectional trisynaptic pathway that constitutes the primary intrinsic circuitry of the hippocampal formation; PRC, perirhinal cortex. 
play a role in mediating the observed relation between anosognosia and dementia severity.

Other features of the prosubiculum that support a putative role in self directed awareness are dense reciprocal connections with the anterior cingulate, ${ }^{18}$ which has been implicated in error detection, ${ }^{19}$ self reflection,,$^{20}$ and awareness of one's actions, ${ }^{21}$ and its dense cholinergic innervation. ${ }^{22}$ Emerging data support a prominent role of acetylcholine in mediating conscious awareness, ${ }^{23}$ and we have reported preliminary findings that increased awareness of personally and environmentally relevant information is among the most consistent effects of cholinesterase inhibitor treatment in patients with Alzheimer's disease. ${ }^{24}{ }^{25}$ Our findings from this study are consistent with a cholinergic component of impaired deficit awareness, as senile plaque density has been reported to be much more robustly correlated with loss of cholinergic markers in Alzheimer's disease than NFT density. ${ }^{12}$ Although we did not observe any histopathological differences between +Aware and -Aware subjects in the medial frontal cortex, this region is noteworthy for having a more variable relation between cholinergic deficits and histopathological burden in Alzheimer's disease. ${ }^{26}$ It is possible that disproportionately severe cholinergic deficits in this area may contribute to altered awareness in Alzheimer's disease.

The current study has several limitations. First, awareness was clinically assessed at an earlier time point before death (on average, two years before death and pathological evaluation) and was based on a derived categorical measure. The lag interval between clinical assessment and death is a common problem in clinicopathological correlation studies and might be addressed by in vivo functional imaging techniques that offer real time correlative data. Although functional brain imaging methods have relatively limited spatial resolution compared with pathological assessment, they offer the potential advantage of being able to assess functional connectivity patterns associated with neural networks involved in generating and modulating different components of awareness. Prospective studies using a validated and graded assessment of awareness would provide a more robust approach to investigating pathological correlates of anosognosia in Alzheimer's disease. This point underscores the variable manifestations of anosognosia in this condition and the need to specify with more precision the nature of the awareness deficit. ${ }^{27}$

Second, although senile plaque counts included both diffuse and neuritic plaques, the fact that all subjects had clinical dementia in the absence of other relevant pathological substrates suggests that Alzheimer's disease was the principal cause of the dementia. Potential subjects with evidence of other pathological lesions were rigorously excluded from this study for the sake of homogeneity. Future studies using more contemporary pathological criteria (NIA-Reagan), modern immunostaining methods, and quantitative stereological techniques would enhance clinicopathological correlation studies.

Third, a relatively limited number of brain regions was assessed, based on tissue availability from this retrospective sample. Different components of anosognosia (for example, awareness of deficit $v$ concern about deficit) may have overlapping but distinctive pathological correlates that could not be examined in this study. Although our findings suggest that amyloid plaque burden in the right prosubiculum region is associated with impaired awareness in Alzheimer's disease, awareness is not an "all or nothing" phenomenon and entails multiple component processes and substrates. Among the few - Aware individuals who had right prosubiculum senile plaque counts below the group mean, the counts tended to be higher in either the right prefrontal (SMF) or temporal (ST) regions. This observation suggests that lesions of the right prosubiculum are a common, though not invariable, correlate of anosognosia in Alzheimer's disease, and that awareness is probably mediated by distributed neural networks. Future studies should include examination of the amygdala, as well as other prefrontal and parietal cortical and subcortical (thalamus) regions, to evaluate possible histopathological differences in other candidate regions that may contribute to anosognosia in Alzheimer's disease.

One of the more intriguing clinical aspects of anosognosia in Alzheimer's disease is its variability. In a cross sectional study of 350 patients with this disorder (MMSE range 5 to 27), $18 \%$ had no insight into their cognitive deficits, $15 \%$ had preserved insight, and the rest had partial awareness of their cognitive deficits. ${ }^{28}$ A recent study by Santillan and colleagues $^{29}$ observed a similar distribution of anosognosia in subjects with Alzheimer's disease, and found that impaired insight was an independent predictor of functional decline. Another recent study ${ }^{30}$ reported apathy to be more strongly associated with anosognosia in subjects with Alzheimer's disease than executive cognitive deficits. Although impaired deficit awareness could be a predisposing factor to greater risk taking behaviour and poor judgment, there is remarkably little information on the natural history and clinical consequences of anosognosia in Alzheimer's disease. Future studies should also address these areas of inquiry, particularly in the earliest disease stages.

\section{ACKNOWLEDGEMENTS}

Supported by US National Institute on Aging Center grant AG0533. We are indebted to the late Dr A Julio Martinez and Dr John Moossy who, along with Dr Gutti R. Rao, were the investigators in the early studies which used the above neuropathological data. We also owe our gratitude to Chad Karoleski who helped in gathering various data from the Alzheimer's Disease Research Center database.

\section{Authors' affiliations}

G A Marshall, S T DeKosky, Department of Neurology, University of Pittsburgh and Pittsburgh VA Medical Center, Pittsburgh, Pennsylvania, USA

G R Rao, R L Hamilton, Department of Pathology (Neuropathology), University of Pittsburgh and Pittsburgh VA Medical Center

O L Lopez, S T DeKosky, Alzheimer's Disease Research Center, University of Pittsburgh and Pittsburgh VA Medical Center

D I Kaufer, Department of Neurology, University of North Carolina, Chapel Hill, North Carolina, USA

Competing interests: none declared

\section{REFERENCES}

1 Lopez OL, Becker JT, Somsak D, et al. Awareness of cognitive deficits and anosognosia in probable Alzheimer's disease. Eur Neurol 1994;34:277-82.

2 Michon A, Deweer B, Pillon B, et al. Relation of anosognosia to frontal lobe dysfunction in Alzheimer's disease. J Neurol Neurosurg Psychiatry 1994;57:805-9.

3 Ott BR, Lafleche G, Whelihan WM, et al. Impaired awareness of deficits in Alzheimer disease. Alzheimer Dis Assoc Disord 1996;10:68-76.

4 Migliorelli R, Teson A, Sabe L, et al. Anosognosia in Alzheimer's disease: a study of associated factors. J Neuropsychiatry Clin Neurosci 1995;7:338-44.

5 Starkstein SE, Sabe L, Chemerinski E, et al. Two domains of anosognosia in Alzheimer's disease. J Neurol Neurosurg Psychiatry 1996;61:485-90.

6 Starkstein SE, Chemerinski E, Sabe L, et al. Prospective longitudinal study of depression and anosognosia in Alzheimer's disease. Br J Psychiatry 1997; 171:47-52.

7 Starkstein SE, Vazquez S, Migliorelli R, et al. A single-photon emission computed tomographic study of anosognosia in Alzheimer's disease. Arch Neurol 1995;52:415-20.

8 Reed BR, Jagust WJ, Coulter L. Anosognosia in Alzheimer's disease: relationships to depression, cognitive function, and cerebral perfusion. J Clin Exp Neuropsychol 1993;15:231-44.

9 Ott BR, Noto RB, Fogel BS. Apathy and loss of insight in Alzheimer's disease: a SPECT imaging study. J Neuropsychiatry Clin Neurosci 1996;8:41-6.

10 Folstein MF, Folstein SE, McHugh PR. "Mini-mental state". A practical method for grading the cognitive state of patients for the clinician. J Psychiatr Res 1975; 12:189-98.

11 Moossy J, Zubenko GS, Martinez AJ, et al. Bilateral symmetry of morphologic lesions in Alzheimer's disease. Arch Neurol 1988;45:251-4. 
12 Zubenko GS, Moossy J, Martinez AJ, et al. A brain regional analysis of morphologic and cholinergic abnormalities in Alzheimer's disease. Arch Neurol 1989;46:634-8.

13 Khachaturian ZS. Diagnosis of Alzheimer's disease. Arch Neurol 1985:42:1097-105.

14 Anonymous. Consensus recommendations for the postmortem diagnosis of Alzheimer's disease. The National Institute on Aging, and Reagan Institute working group on diagnostic criteria for the neuropathological assessment of Alzheimer's disease. Neurobiol Aging 1997;18(4 suppl):S1-2.

15 Amaral DG. Memory: anatomical organization of candidate brain regions. In: Mountcastle VB, Plum F, eds. Handbook of neurophysiology. The nervous system, vol V. Bethesda: American Physiological Society, 1987:211-89.

16 Hyman BT, Van Hoesen GW, Damasio AR. Memory-related neural systems in Alzheimer's disease: an anatomic study. Neurology 1990;40:1721-30.

17 Turner BH, Knapp ME. Consciousness: a neurobiological approach. Integr Physiol Behav Sci 1995;30:151-6.

18 Arikuni T, Sako H, Murata A. Ipsilateral connections of the anterior cingulate cortex with the frontal and medial temporal cortices in the macaque monkey. Neurosci Res 1994:21:19-39.

19 Carter CS, Braver TS, Barch DM, et al. Anterior cingulate cortex, error detection, and the online monitoring of performance. Science 1998;280:747-9.

20 Johnson SC, Baxter LC, Wilder LS, et al. Neural correlates of self-reflection. Brain 2002;125:1808-14.

21 Frith C. Attention to action and awareness of other minds. Conscious Cogn 2002;11:481-7.
22 Green RC, Mesulam MM. Acetylcholinesterase fiber staining in the human hippocampus and parahippocampal gyrus. J Comp Neurol 1988;273:488-99.

23 Perry E, Walker M, Grace J, et al. Acetylcholine in mind: a neurotransmitter correlate of consciousness? Trends Neurosci 1999;22:273-80.

24 Kaufer DI. Alzheimer's disease: focus on the cholinergic system. In: Perry E, Ashton $\mathrm{H}$, Young A, eds. Neurochemistry of consciousness, neurotransmitters in mind. Amsterdam: John Benjamins, 2001:229-45.

25 Kaufer DI. Cholinesterase-inhibitor therapy in dementia: novel clinical substrates and mechanisms of treatment response. CNS Spectrums 2002;7:742-50.

26 Geula C. Abnormalities of neural circuitry in Alzheimer's disease: hippocampus and cortical cholinergic innervation. Neurology 1998:51(suppl 1):S18-29.

27 Agnew SK, Morris RG. The heterogeneity of anosognosia for memory impairment in Alzheimer's disease: a review of the literature and a proposed model. Aging Mental Health 1998;2:7-19.

28 Morris JC, Heyman A, Mohs RC, et al. The Consortium to Establish a Registry for Alzheimer's Disease (CERAD). Part I. Clinical and neuropsychological assessment of Alzheimer's disease. Neurology 1989;39:1159-65.

29 Santillan CE, Fritsch T, Geldmacher DS. Development of a scale to predict decline in patients with mild Alzheimer's disease. J Am Geriatr Soc 2003;51:91-5.

30 Derouesne C, Thibault S, Lagha-Pierucci S, et al. Decreased awareness of cognitive deficits in patients with mild dementia of the Alzheimer type. Int J Geriatr Psychiatry 1999;14:1019-30. 